\title{
A STOCHASTIC TIME-DELAYED MODEL FOR THE EFFECTIVENESS OF MOROCCAN COVID-19 DECONFINEMENT STRATEGY*,**
}

\author{
Houssine Zine ${ }^{1}$, Adnane Boukhouima ${ }^{2}$, El Mehdi Lotfi², \\ Marouane Mahrouf ${ }^{2}$, Delfim F.M. Torres ${ }^{1, * * *}$ And Noura Yousfi ${ }^{2}$
}

\begin{abstract}
Coronavirus disease 2019 (COVID-19) poses a great threat to public health and the economy worldwide. Currently, COVID-19 evolves in many countries to a second stage, characterized by the need for the liberation of the economy and relaxation of the human psychological effects. To this end, numerous countries decided to implement adequate deconfinement strategies. After the first prolongation of the established confinement, Morocco moves to the deconfinement stage on May 20, 2020. The relevant question concerns the impact on the COVID-19 propagation by considering an additional degree of realism related to stochastic noises due to the effectiveness level of the adapted measures. In this paper, we propose a delayed stochastic mathematical model to predict the epidemiological trend of COVID-19 in Morocco after the deconfinement. To ensure the well-posedness of the model, we prove the existence and uniqueness of a positive solution. Based on the large number theorem for martingales, we discuss the extinction of the disease under an appropriate threshold parameter. Moreover, numerical simulations are performed in order to test the efficiency of the deconfinement strategies chosen by the Moroccan authorities to help the policy makers and public health administration to make suitable decisions in the near future.
\end{abstract}

Mathematics Subject Classification. 60H10, 92D30.

Received May 16, 2020. Accepted October 28, 2020.

\section{INTRODUCTION}

Coronavirus disease 2019 (COVID-19), reclassified as a pandemic by the World Health Organization (WHO) on March 11, 2020 [14], is an infectious disease caused by a new type of virus belonging to the coronaviruses family and recently named severe acute respiratory syndrome coronavirus 2 (SARS-CoV-2) [4]. All the countries affected by this disease have taken many preventive measures, including containment. The containment established by

\footnotetext{
* This research is part of first author's Ph.D. project, which is carried out at University of Aveiro.

** Work partially supported through FCT, project UIDB/04106/2020 (CIDMA).

Keywords and phrases: Coronavirus disease 2019 (COVID-19), deconfinement strategy, mathematical modeling, delayed stochastic differential equations (DSDEs), extinction.

${ }^{1}$ Center for Research and Development in Mathematics and Applications (CIDMA), Department of Mathematics, University of Aveiro, 3810-193 Aveiro, Portugal.

${ }^{2}$ Laboratory of Analysis, Modeling and Simulation (LAMS), Faculty of Sciences Ben M'sik, Hassan II University of Casablanca, P.B 7955 Sidi Othman, Casablanca, Morocco.

*** Corresponding author: delfim@ua.pt
} 
the Moroccan government and the public authorities at the right time made it possible to avoid the worst: according to the minister of Health, at least 6000 lives were saved thanks to the measures adopted to face the spread of this pandemic [13]. The resistance measures, regarded as necessary and urgent, cannot be sustainable.

Actually, the deconfinement is a new stage entered by the COVID-19 pandemic. Therefore, several countries strategically planned their deconfinement strategies. The extension of the state of emergency in Morocco until May 20, 2020 will no doubt have economic repercussions. If Morocco won the first round, or at least limited the consequences, especially in terms of limiting the pandemic and health management of the situation, the second seems difficult and complex. Indeed, it must not only be well thought out but also its axes of resistance have to be well-identified. In this context, all efforts should be focused on stabilizing the economy by intelligently relying on resources. Economic deconfinement is part of the solution and should be gradual and concerted. Indeed, it is absurd to think that the return to the normality is in the near months, because the unavailability of an effective vaccine implies that the virus will always be with us in the near future, which poses a risk for the population. This economic deconfinement should be prepared and accompanied by other related measures, in particular under health, security, education and social assistance. In this period of general crisis, the response must try to mitigate the impacts on priority sectors, such as agriculture, agrifood, transport and foreign trade, in relation to imports that are vital to the Moroccan economy. The challenge is to ensure resistance and a continuity of value creation while preventing a sector from being detached from the economic body. So to speak, priority must be given to vital sectors whose health directly affects all Moroccan activity, while protecting those bordering on chaos. According to the deconfinement strategy, which is applied by the Moroccan authorities, it is mandatory to study the occurrence of an eventual second wave and it's magnitude.

Mathematical modeling through dynamical systems plays an important role to predict the evolution of COVID-19 transmission $[9,16]$. However, while taking into account the deconfinement policies, the environmental effects and the social fluctuations should not be neglected in such a mathematical study in order to describe well the dynamics and consider an additional degree of realism $[2,3,10,18,21]$. For these reasons, we describe here the dynamics of the deconfinement strategy by a new D-COVID-19 model, governed by delayed stochastic differential equations (DSDE), as follows:

$$
\left\{\begin{aligned}
\mathrm{d} S(t)= & \left(\rho C(t)-\delta S(t)-\beta(1-u) \frac{S(t) I_{s}(t)}{N}\right) \mathrm{d} t-\sigma_{1}(1-u) \frac{S(t) I_{s}(t)}{N} \mathrm{~d} B_{1}(t)+\sigma_{2}(C(t)-S(t)) \mathrm{d} B_{2}(t), \\
\mathrm{d} C(t)= & (\delta S(t)-\rho C(t)) \mathrm{d} t+\sigma_{2}(S(t)-C(t)) \mathrm{d} B_{2}(t), \\
\mathrm{d} I_{s}(t)= & \left(\beta \epsilon(1-u) \frac{S\left(t-\tau_{1}\right) I_{s}\left(t-\tau_{1}\right)}{N}-\alpha I_{s}(t)-(1-\alpha)\left(\mu_{s}+\eta_{s}\right) I_{s}(t)\right) \mathrm{d} t \\
& +\sigma_{1}\left(\epsilon(1-u) \frac{S\left(t-\tau_{1}\right) I_{s}\left(t-\tau_{1}\right)}{N}\right) \mathrm{d} B_{1}(t)+\sigma_{3}\left(\mu_{s}+\eta_{s}-1\right) I_{s}(t) \mathrm{d} B_{3}(t), \\
\mathrm{d} I_{a}(t)= & \left(\beta(1-\epsilon)(1-u) \frac{S\left(t-\tau_{1}\right) I_{s}\left(t-\tau_{1}\right)}{N}-\eta_{a} I_{a}(t)\right) \mathrm{d} t+\sigma_{1}(1-\epsilon)(1-u) \frac{S\left(t-\tau_{1}\right) I_{s}\left(t-\tau_{1}\right)}{N} \mathrm{~d} B_{1}(t), \\
\mathrm{d} F_{b}(t)= & \left(\alpha \gamma_{b} I_{s}\left(t-\tau_{2}\right)-\left(\mu_{b}+r_{b}\right) F_{b}(t)\right) \mathrm{d} t+\sigma_{3} \gamma_{b} I_{s}\left(t-\tau_{2}\right) \mathrm{d} B_{3}(t), \\
\mathrm{d} F_{g}(t)= & \left(\alpha \gamma_{g} I_{s}\left(t-\tau_{2}\right)-\left(\mu_{g}+r_{g}\right) F_{g}(t)\right) \mathrm{d} t+\sigma_{3} \gamma_{g} I_{s}\left(t-\tau_{2}\right) \mathrm{d} B_{3}(t), \\
\mathrm{d} F_{c}(t)= & \left(\alpha \gamma_{c} I_{s}\left(t-\tau_{2}\right)-\left(\mu_{c}+r_{c}\right) F_{c}(t)\right) \mathrm{d} t+\sigma_{3} \gamma_{c} I_{s}\left(t-\tau_{2}\right) \mathrm{d} B_{3}(t), \\
\mathrm{d} R(t)= & \left(\eta_{s}(1-\alpha) I_{s}\left(t-\tau_{3}\right)+\eta_{a} I_{a}\left(t-\tau_{3}\right)+r_{b} F_{b}\left(t-\tau_{4}\right)+r_{g} F_{g}\left(t-\tau_{4}\right)+r_{c} F_{c}\left(t-\tau_{4}\right)\right) \mathrm{d} t \\
& -\sigma_{3} \eta_{s} I_{s}\left(t-\tau_{3}\right) \mathrm{d} B_{3}(t), \\
\mathrm{d} M(t)= & \left(\mu_{s}(1-\alpha) I_{s}\left(t-\tau_{3}\right)+\mu_{b} F_{b}\left(t-\tau_{4}\right)+\mu_{g} F_{g}\left(t-\tau_{4}\right)+\mu_{c} F_{c}\left(t-\tau_{4}\right)\right) \mathrm{d} t-\sigma_{3} \mu_{s} I_{s}\left(t-\tau_{3}\right) \mathrm{d} B_{3}(t),
\end{aligned}\right.
$$




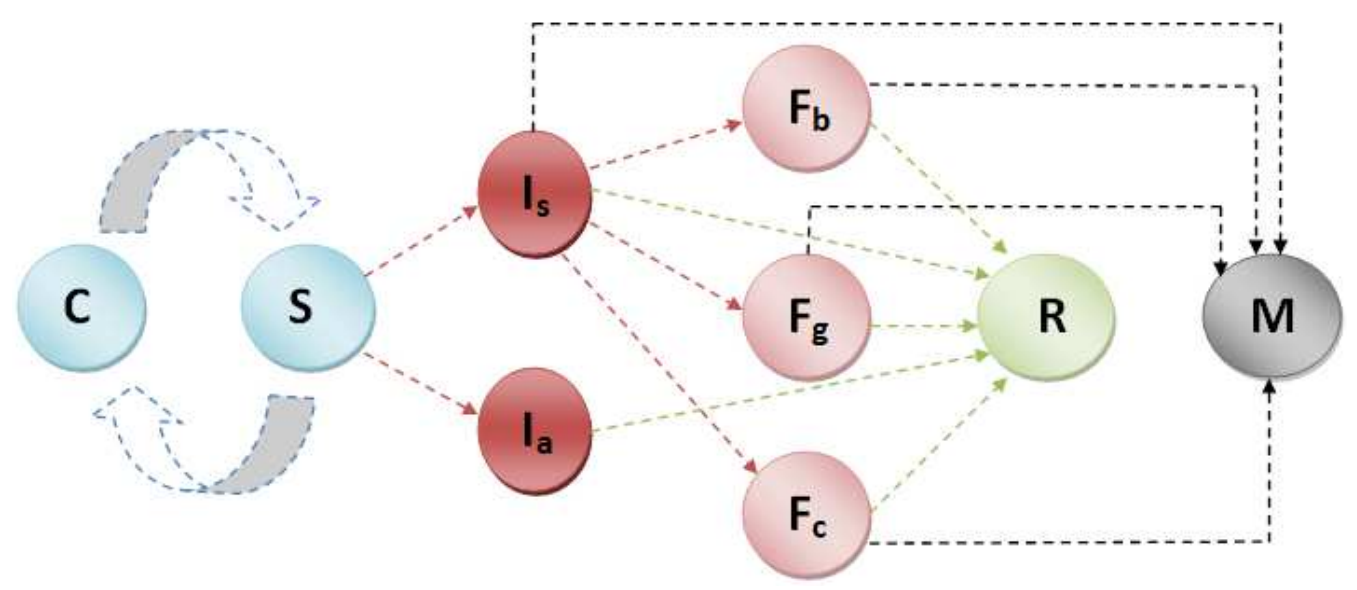

FiguRE 1. Schematic diagram of model (1.1).

where $S$ represents the susceptible sub-population, which is not infected and has not been infected before but is susceptible to develop the disease if exposed to the virus; $C$ is the confined sub-population; $I_{s}$ is the symptomatic infected sub-population, which has not yet been treated, it transmits the disease, and outside of proper support it can progress to spontaneous recovery or death; $I_{a}$ is the asymptomatic infected sub-population who is infected but does not transmit the disease, is not known by the health system and progresses spontaneously to recovery; $F_{b}, F_{g}$ and $F_{c}$ are the patients diagnosed, supported by the Moroccan health system and under quarantine, and subdivided into three categories: benign, severe, critical forms, respectively. Finally, $R$ and $M$ are the recovered and died classes, respectively. At each instant of time, the equation

$$
D(t):=\mu_{s}(1-\alpha) I_{s}\left(t-\tau_{3}\right)+\mu_{b} F_{b}\left(t-\tau_{4}\right)+\mu_{g} F_{g}\left(t-\tau_{4}\right)+\mu_{c} F_{c}\left(t-\tau_{4}\right)-\sigma_{3} \mu_{s} I_{s}\left(t-\tau_{3}\right) \frac{\Delta B_{3}(t)}{\Delta t}=\frac{\Delta M(t)}{\Delta t}
$$

gives the number of the new dead due to disease. The parameter $1-u$ represents the level of measures undertaken on the susceptible population while $\delta$ is the confinement rate and $\rho$ represents the deconfinement rate. We adopt the bilinear incidence rate to describe the infection of the disease and use the parameter $\beta$ to denote the transmission rate. It is reasonable to assume that the infected individuals are subdivided into individuals with symptoms and others without symptoms, for which we employ the parameter $\epsilon$ to denote the proportion for the symptomatic individuals and $1-\epsilon$ for the asymptomatic ones. The parameter $\alpha$ measures the efficiency of public health administration for hospitalization. Diagnosed symptomatic infected population is completely distributed into one of the three forms $F_{b}, F_{g}$ and $F_{c}$, by the rates $\gamma_{b}, \gamma_{g}$ and $\gamma_{c}$, respectively. Then, $\gamma_{b}+\gamma_{g}+\gamma_{c}=1$. The mean recovery period of these forms are denoted by $1 / r_{b}, 1 / r_{g}$ and $1 / r_{c}$, respectively. The latter forms die also with the rates $\mu_{b}, \mu_{g}$ and $\mu_{c}$, respectively. Symptomatic infected population, which is not diagnosed, moves to the recovery compartment with a rate $\eta_{s}$ or dies with a rate $\mu_{s}$. On the other hand, asymptomatic infected population moves to the recovery compartment with a rate $\eta_{a}$. The time delays $\tau_{1}$ and $\tau_{2}$ denote the incubation period and the period of time needed before the charge by the health system, respectively. The time delays $\tau_{3}$ and $\tau_{4}$ denote the time required before the death of individuals coming from the compartments $I_{s}$ and the three forms $F_{b}, F_{g}$ and $F_{c}$, respectively. Here, $B_{1}(t), B_{2}(t)$ and $B_{3}(t)$ are independent standard Brownian motions defined on a complete probability space $(\Omega, \mathcal{F}, \mathbb{P})$ with a filtration $\left\{\mathcal{F}_{t}\right\}_{t>0}$ and satisfying the usual conditions, that is, they are increasing and right continuous while $\mathcal{F}_{0}$ contains all P-null sets and $\sigma_{i}$ represents the intensity of $B_{i}, i=1,2,3$. The schematic diagram of our extended model is illustrated in Figure 1.

Remark 1.1. The stochasticity is introduced in model (1.1) by perturbing the most sensitive parameters: $\beta$, $\alpha, \delta$, and $\rho$. 
Remark 1.2. For the sake of simplicity, we have assumed that the parameters $\delta$ and $\rho$ are perturbed with the same intensities, that is, we assume that Moroccan individuals possess the same behaviors and reactions towards the authorities instructions.

Remark 1.3. Note that the multipliers of $I_{s}\left(t-\tau_{2}\right)$ terms are the same as $\sigma_{3} \mathrm{~d} B_{3}(t)$ although they are premultiplied by different constants. Indeed, the portion of diagnosed symptomatic infected population is completely distributed into the three forms $F_{b}, F_{g}$, and $F_{c}$, by the rates $\gamma_{b}, \gamma_{g}$ and $\gamma_{c}$, respectively. Then, $\gamma_{b}+\gamma_{g}+\gamma_{c}=1$. In addition, we assume that the parameter $\alpha$, which measures the efficiency of public health administration for hospitalization, undergoes random fluctuations.

Remark 1.4. For illustration and clarification purposes, let us suppose, as an example, that the disease progression is started from 15th of March and value of $\tau_{1}$ is 5 . Then a susceptible individual, after contact with an infected one at instant $t$, becomes himself infected at instant $t+\tau_{1}$. Suddenly, the compartment of the infected is fed at the instant $t$ by the susceptible infected at the instant $t-\tau_{1}$. Therefore, in the considered situation, when the infection starts at March 15, the term $\beta \epsilon(1-u) S(T) I_{s}(T) / N$ of new infected is equal to zero on 12 th, 13th and 14th March, due to the absence of the infection.

Remark 1.5. Temporarily asymptomatic individuals are included in the class $I_{s}$ of symptomatic, while individuals in $I_{a}$, who are permanently asymptomatic, will remain asymptomatic until recovery and will not spread the virus, a fact which has been recently confirmed by the World Health Organization.

For biological reasons, we assume that the initial conditions of system (1.1) satisfy:

$$
\begin{aligned}
& S(\theta)=\phi_{1}(\theta) \geq 0, \quad C(\theta)=\phi_{2}(\theta) \geq 0, \quad I_{a}(\theta)=\phi_{3}(\theta) \geq 0, \\
& I_{s}(\theta)=\phi_{4}(\theta) \geq 0, \quad F_{b}(\theta)=\phi_{5}(\theta) \geq 0, \quad F_{g}(\theta)=\phi_{6}(\theta) \geq 0, \\
& F_{c}(\theta)=\phi_{7}(\theta) \geq 0, \quad R(\theta)=\phi_{8}(\theta) \geq 0, \quad M(\theta)=\phi_{9}(\theta) \geq 0,
\end{aligned}
$$

where $\theta \in[-\tau, 0]$ and $\tau=\max \left\{\tau_{1}, \tau_{2} \tau_{3}, \tau_{4}\right\}$.

The rest of the paper is organized as follows. Section 2 deals with the existence and uniqueness of a positive global solution that ensures the well-posedness of the D-COVID-19 model (1.1). A sufficient condition for the extinction is established in Section 3. Then, some numerical scenarios, to assess the effectiveness of the adopted deconfinement strategy, are presented in Section 4. The paper ends up with Section 5 of conclusion.

\section{Existence And uniqueness of a Positive Global SOlution}

Let us denote $\mathbb{R}_{+}^{9}:=\left\{\left(x_{1}, x_{2}, x_{3}, x_{4}, x_{5}, x_{6}, x_{7}, x_{8}, x_{9}\right) \mid x_{i}>0, i=1,2, \ldots, 9\right\}$. We begin by proving the following result.

Theorem 2.1. For any initial value satisfying condition (1.2), there is a unique solution

$$
x(t)=\left(S(t), C(t), I_{s}(t), I_{a}(t), F_{b}(t), F_{g}(t), F_{c}(t), R(t), M(t)\right)
$$

to the D-COVID-19 model (1.1) that remains in $\mathbb{R}_{+}^{9}$ with probability one.

Proof. Since the coefficients of the Stochastic Differential Equations with several delays (1.1) are locally Lipschitz continuous, it follows from [12] that for any square integrable initial value $x(0) \in \mathbb{R}_{+}^{9}$, which is independent of the considered standard Brownian motion $B$, there exists a unique local solution $x(t)$ on $t \in\left[0, \tau_{e}\right)$, where $\tau_{e}$ is the explosion time. For showing that this solution is global, knowing that the linear growth condition is not verified, we need to prove that $\tau_{e}=\infty$. Let $k_{0}>0$ be sufficiently large for $\frac{1}{k_{0}}<x(0)<k_{0}$. For each integer $k \geq k_{0}$, we define the stopping time $\tau_{k}:=\inf \left\{t \in\left[0, \tau_{e}\right) / x_{i}(t) \notin\left(\frac{1}{k}, k\right)\right.$ for some $\left.i=1,2,3\right\}$, where $\inf \emptyset=\infty$. 
It is evident that $\tau_{k} \leq \tau_{e}$. Let $T>0$, and define the twice differentiable function $V$ on $\mathbb{R}_{+}^{3} \rightarrow \mathbb{R}^{+}$as follows:

$$
V(x):=\left(x_{1}+x_{2}+x_{3}\right)^{2}+\frac{1}{x_{1}}+\frac{1}{x_{2}}+\frac{1}{x_{3}} .
$$

By Itô's formula, for any $0 \leq t \leq \tau_{k} \wedge T$ and $k \geq 1$ we have

$$
d V(x(t))=L V(x(t)) \mathrm{d} t+\sigma(x(t)) \mathrm{d} B_{t},
$$

where $L$ is the differential operator of function $V$ :

$$
\begin{aligned}
L V(x(t))= & \left(2\left(S(t)+C(t)+I_{s}(t)\right)-\frac{1}{S^{2}(t)}\right)\left(\rho C(t)-\delta S(t)-\beta(1-u) \frac{S(t) I_{s}(t)}{N}\right) \\
& +\frac{1}{2}\left(2+\frac{2}{S^{3}(t)}\right)\left(\left(-\sigma_{1}(1-u) \frac{S(t) I_{s}(t)}{N}\right)^{2}+\left(\sigma_{2}(C(t)-S(t))\right)^{2}\right) \\
& +\left(2\left(S(t)+C(t)+I_{s}(t)\right)-\frac{1}{C^{2}(t)}\right)(\delta S(t)-\rho C(t))+\frac{1}{2}\left(2+\frac{2}{C^{3}(t)}\right)\left(-\sigma_{2}(C(t)-S(t))\right)^{2} \\
& +\frac{1}{2}\left(2+\frac{2}{C^{3}(t)}\right)\left(-\sigma_{2}(C(t)-S(t))\right)^{2} \\
& +\left(2\left(S(t)+C(t)+I_{s}(t)\right)-\frac{1}{I_{s}^{2}(t)}\right)\left(\beta \epsilon(1-u) \frac{S\left(t-\tau_{1}\right) I_{s}\left(t-\tau_{1}\right)}{N}-\alpha I_{s}(t)-(1-\alpha)\left(\mu_{s}+\eta_{s}\right) I_{s}(t)\right) \\
& +\frac{1}{2}\left(2+\frac{2}{I_{s}^{3}(t)}\right)\left(\left(-\sigma_{3} I_{s}(t)\left(1-\mu_{s}-\eta_{s}\right)\right)^{2}+\left(\sigma_{1} \epsilon(1-u) \frac{S\left(t-\tau_{1}\right) I_{s}\left(t-\tau_{1}\right)}{N}\right)^{2}\right) .
\end{aligned}
$$

Thus,

$$
\begin{aligned}
L V(x(t)) \leq & 2\left(S(t)+C(t)+I_{s}(t)\right) \rho C(t)+\frac{\delta}{S(t)}+\frac{\beta(1-u) S(t) I_{s}(t)}{N S^{2}(t)} \\
& +\left(1+\frac{1}{S^{3}(t)}\right)\left(\left(\sigma_{1}(1-u) \frac{S(t) I_{s}(t)}{N}\right)^{2}+\left(\sigma_{2}(C(t)-S(t))\right)^{2}\right) \\
& +2\left(S(t)+C(t)+I_{s}(t)\right) \delta S(t)+\frac{\rho}{S(t)}+\left(1+\frac{1}{C^{3}(t)}\right)\left(\sigma_{2}(C(t)-S(t))\right)^{2} \\
& +2\left(S(t)+C(t)+I_{s}(t)\right) \beta \epsilon(1-u) \frac{S\left(t-\tau_{1}\right) I_{s}\left(t-\tau_{1}\right)}{N} \\
& +\frac{\alpha}{I_{s}(t)}+(1-\alpha)\left(\mu_{s}+\eta_{s}\right) \frac{1}{I_{s}(t)} \\
& +\left(1+\frac{1}{I_{s}^{3}(t)}\right)\left(\left(-\sigma_{3} I_{s}(t)\left(1-\mu_{s}-\eta_{s}\right)^{2}+\left(\sigma_{1} \epsilon(1-u) \frac{S\left(t-\tau_{1}\right) I_{s}\left(t-\tau_{1}\right)}{N}\right)^{2}\right) .\right.
\end{aligned}
$$

By applying the elementary inequality $2 a b \leq a^{2}+b^{2}$, we can easily increase the right-hand side of the previous inequality to obtain that

$$
L V(x) \leq D(1+V(x))
$$


where $D$ is an adequate selected positive constant. By integrating both sides of the equality

$$
d V(x(t))=L V(x(t)) \mathrm{d} t+\sigma(x(t)) \mathrm{d} B_{t}
$$

between $t_{0}$ and $t \wedge \tau_{k}$ and acting the expectation, which eliminates the martingale part, we get that

$$
\begin{aligned}
E\left(V\left(x\left(t \wedge \tau_{k}\right)\right)\right. & \left.=E\left(V\left(x_{0}\right)\right)+E \int_{t_{0}}^{t \wedge \tau_{k}} L V\left(x_{s}\right)\right) \mathrm{d} s \\
& \leq E\left(V\left(x_{0}\right)\right)+E \int_{t_{0}}^{t \wedge \tau_{k}} D\left(1+V\left(x_{s}\right)\right) \mathrm{d} s \\
& \left.\leq E\left(V\left(x_{0}\right)\right)+D T+\int_{t_{0}}^{t \wedge \tau_{k}} E V\left(x_{s}\right)\right) \mathrm{d} s .
\end{aligned}
$$

Gronwall's inequality implies that

$$
E\left(V\left(x\left(t \wedge \tau_{k}\right)\right) \leq\left(E V\left(x_{0}\right)+D T\right) \exp (C T)\right.
$$

For $\omega \in\left\{\tau_{k} \leq T\right\}, x_{i}\left(\tau_{k}\right)$ equals $k$ or $\frac{1}{k}$ for some $i=1,2,3$. Hence,

$$
V\left(x_{i}\left(\tau_{k}\right)\right) \geq\left(k^{2}+\frac{1}{k}\right) \wedge\left(\frac{1}{k^{2}}+k\right) .
$$

It follows that

$$
\begin{aligned}
\left(E V\left(x_{0}\right)+D T\right) \exp (C T) & \geq E\left(\chi_{\left\{\tau_{k} \leq T\right\}}(\omega) V\left(x_{\tau_{k}}\right)\right) \\
& \geq\left(k^{2}+\frac{1}{k}\right) \wedge\left(\frac{1}{k^{2}}+k\right) P\left(\tau_{k} \leq T\right) .
\end{aligned}
$$

Letting $k \rightarrow \infty$, we get $P\left(\tau_{e} \leq T\right)=0$. Since $T$ is arbitrary, we obtain $P\left(\tau_{e}=\infty\right)=1$. With the same technique, we also deduce that the rest of the variables of the system are positive on $[0, \infty)$. This concludes the proof.

\section{Extinction OF THE Disease}

In this section, we obtain a sufficient condition for the extinction of the disease.

Theorem 3.1. Let $\left(S(t), C(t), I_{s}(t), I_{a}(t), F_{b}(t), F_{g}(t), F_{c}(t), R(t), M(t)\right)$ be a solution of the D-COVID-19 model (1.1) with positive initial value defined in (1.2). Assume that

$$
\sigma_{1}^{2}>\frac{\beta^{2}}{2\left(\alpha+(1-\alpha)\left(\mu_{s}+\eta_{s}\right)\right)} .
$$

Then,

$$
\limsup _{t \rightarrow \infty} \ln \frac{I_{s}(t)}{t}<0
$$

Namely, $I_{s}(t)$ tends to zero exponentially a.s., that is, the disease dies out with probability 1. 
Proof. Let

$$
\begin{aligned}
d \ln I_{s}(t)= & {\left[\frac{1}{I_{s}(t)}\left(\frac{\beta \epsilon(1-u) S\left(t-\tau_{1}\right) I_{s}\left(t-\tau_{1}\right)}{N}-\alpha I_{s}(t)-(1-\alpha)\left(\mu_{s}+\eta_{s}\right) I_{s}(t)\right)\right.} \\
& \left.-\frac{1}{2 I_{s}^{2}(t)}\left(\left(\sigma_{1} \frac{\beta \epsilon(1-u) S\left(t-\tau_{1}\right) I_{s}\left(t-\tau_{1}\right)}{N}\right)^{2}+\left(\sigma_{3}\left(\mu_{s}+\eta_{s}-1\right) I_{s}(t)\right)^{2}\right)\right] \mathrm{d} t \\
& +\frac{1}{I_{s}(t)} \sigma_{1} \frac{\beta \epsilon(1-u) S\left(t-\tau_{1}\right) I_{s}\left(t-\tau_{1}\right)}{N} \mathrm{~d} B_{1}+\frac{1}{I_{s}(t)} \sigma_{3}\left(\mu_{s}+\eta_{s}-1\right) I_{s}(t) \mathrm{d} B_{3} .
\end{aligned}
$$

To simplify, we set

$$
\begin{aligned}
G(t) & :=\frac{\epsilon(1-u) S\left(t-\tau_{1}\right) I_{s}\left(t-\tau_{1}\right)}{N}, \\
R_{1}(t) & :=\frac{\sigma_{1}}{I_{s}(t)} \frac{\beta \epsilon(1-u) S\left(t-\tau_{1}\right) I_{s}\left(t-\tau_{1}\right)}{N}=\frac{\beta \sigma_{1}}{I_{s}(t)} G, \\
R_{3}(t) & :=\frac{\sigma_{3}}{I_{s}(t)}\left(\mu_{s}+\eta_{s}-1\right) I_{s}(t), \\
H & :=-\alpha-(1-\alpha)\left(\mu_{s}+\eta_{s}\right) .
\end{aligned}
$$

We then get

$$
\begin{aligned}
\mathrm{d} \ln I_{s}(t) & =\frac{\beta G(t)}{I_{s}(t)}-H(t)-\frac{1}{2}\left(\left(\frac{\sigma_{1} G(t)}{I_{s}(t)}\right)^{2}+\left(\sigma_{3}\left(\mu_{s}+\eta_{s}-1\right) I_{s}(t)\right)^{2}\right)+R_{1}(t) \mathrm{d} B_{1}+R_{3}(t) \mathrm{d} B_{3} \\
& =-\frac{\sigma_{1}^{2}}{2}\left[\left(\frac{G(t)}{I_{s}(t)}\right)^{2}-\frac{2 \beta}{\sigma_{1}^{2}} \frac{G(t)}{I_{s}(t)}\right]+H+R_{1}(t) \mathrm{d} B_{1}+R_{3}(t) \mathrm{d} B_{3} \\
& =-\frac{\sigma_{1}^{2}}{2}\left[\left(\frac{G(t)}{I_{s}(t)}-\frac{\beta}{\sigma_{1}^{2}}\right)^{2}-\frac{\beta^{2}}{\sigma_{1}^{4}}\right]+H+R_{1}(t) \mathrm{d} B_{1}+R_{3}(t) \mathrm{d} B_{3} \\
& \leq \frac{\beta^{2}}{2 \sigma_{1}^{2}}+H+R_{1}(t) \mathrm{d} B_{1}+R_{3}(t) \mathrm{d} B_{3} .
\end{aligned}
$$

Hence,

$$
\frac{\ln I_{s}(t)}{t} \leq \frac{\ln I_{s}(0)}{t}+\frac{\beta^{2}}{2 \sigma_{1}^{2}}+H+\frac{M_{1}(t)}{t}+\frac{M_{3}(t)}{t}
$$

where

$$
M_{1}(t)=\int_{0}^{t} R_{1}(s) \mathrm{d} B_{1}, \quad M_{3}(t)=\int_{0}^{t} R_{3}(s) \mathrm{d} B_{3} .
$$

We have

$$
\begin{aligned}
\left\langle M_{1}, M_{1}\right\rangle_{t} & =\int_{0}^{t}\left(\frac{1}{I_{s}(s)} \sigma_{1} G(s)\right)^{2} \mathrm{~d} s \\
& =\int_{0}^{t} \sigma_{1}{ }^{2} \epsilon^{2}(1-u)^{2} \frac{S\left(t-\tau_{1}\right)^{2} I_{s}\left(t-\tau_{1}\right)^{2}}{N^{2} I_{s}^{2}} \mathrm{~d} s
\end{aligned}
$$




$$
\begin{aligned}
& \leq \int_{0}^{t} \sigma_{1}{ }^{2} \epsilon^{2}(1-u)^{2} \frac{N^{4}}{N^{2}} \frac{1}{I_{s}^{2}} \mathrm{~d} s \\
& \leq \int_{0}^{t} \sigma_{1}{ }^{2} \epsilon^{2}(1-u)^{2} N^{2} \mathrm{~d} s .
\end{aligned}
$$

Then,

$$
\limsup _{t \rightarrow \infty} \frac{\left\langle M_{1}, M_{1}\right\rangle_{t}}{t} \leq \sigma_{1}^{2} \epsilon^{2}(1-u)^{2} N^{2}<\infty
$$

From the large number theorem for martingales [5], we deduce that

$$
\lim _{t \rightarrow \infty} \frac{M_{1}(t)}{t}=0
$$

We also have

$$
\begin{aligned}
\left\langle M_{2}, M_{2}\right\rangle_{t} & =\int_{0}^{t}\left(\frac{1}{I_{s}(s)} \sigma_{3}\left(\mu_{s}+\eta_{s}-1\right) I_{s}(s)\right)^{2} \mathrm{~d} s \\
& =\int_{0}^{t}\left(\sigma_{3}^{2}\left(\mu_{s}+\eta_{s}-1\right)^{2}\right) \mathrm{d} s \\
& =\left(\sigma_{3}^{2}\left(\mu_{s}+\eta_{s}-1\right)^{2}\right) t .
\end{aligned}
$$

Thus,

$$
\limsup _{t \rightarrow \infty} \frac{\left\langle M_{2}, M_{2}\right\rangle_{t}}{t} \leq \sigma_{3}^{2}\left(\mu_{s}+\eta_{s}-1\right)<\infty
$$

We deduce that

$$
\lim _{t \rightarrow \infty} \frac{M_{2}(t)}{t}=0
$$

Subsequently,

$$
\limsup _{t \rightarrow \infty} \frac{\ln I_{s}(t)}{t} \leq \frac{\beta^{2}}{2 \sigma_{1}^{2}}-\alpha-(1-\alpha)\left(\mu_{s}+\eta_{s}\right)
$$

We conclude that if $\frac{\beta^{2}}{2 \sigma_{1}^{2}}-\alpha-(1-\alpha)\left(\mu_{s}+\eta_{s}\right)<0$, then $\lim _{t \rightarrow \infty} I(t)=0$. The proof is complete. 
TABLE 1. Summary of considered non-pharmaceutical interventions.

\begin{tabular}{ll}
\hline Policies & Decisions made at the government level \\
\hline With minimal social distancing measure & $u=0.1$, on [March 2, March 16] and \\
& $u=0.2$, on (March 16, March 20] \\
With middle social distancing measure & $u=0.1$, on [March 2, March 16], \\
& $u=0.2$, on (March 16, March 20] and \\
& $u=0.4$, on (March 20, April 6] \\
With high social distancing measure & $u=0.1$, on [March 2, March 16], \\
& $u=0.2$ on (March 16, March 20], \\
& $u=0.4$, on (March 20, April 6] and \\
With maximal social distancing measure & $u=0.6$, on (April 6, April 25] \\
& $u=0.1$ on [March 2, March 16], \\
& $u=0.2$, on (March 16, March 20], \\
& $u=0.4$, on (March 20, April 6], \\
& $u=0.6$, on (April 6, April 25] and \\
& $u=0.7$, from April 25 on. \\
\hline
\end{tabular}

TABLE 2. Parameter values for the D-COVID-19 model (1.1).

\begin{tabular}{lccccccccc}
\hline Parameter & $\beta$ & $\epsilon$ & $\gamma_{b}$ & $\gamma_{g}$ & $\gamma_{c}$ & $\alpha$ & $\eta_{a}$ & $\eta_{s}$ & $\mu_{s}$ \\
\hline Value & 0.4517 & 0.794 & 0.8 & 0.15 & 0.05 & 0.06 & $1 / 21$ & $0.8 / 21$ & $0.01 / 21$ \\
\hline
\end{tabular}

\begin{tabular}{lcccccccccc}
\hline Parameter & $\mu_{b}$ & $\mu_{g}$ & $\mu_{c}$ & $r_{b}$ & $r_{g}$ & $r_{c}$ & $\tau_{1}$ & $\tau_{2}$ & $\tau_{3}$ & $\tau_{4}$ \\
\hline Value & 0 & 0 & $0.4 / 13.5$ & $1 / 13.5$ & $1 / 13.5$ & $0.6 / 13.5$ & 5.5 & 7.5 & 21 & 13.5 \\
\hline
\end{tabular}

\section{Results And Discussion}

In this section, we simulate the forecasts of the D-COVID-19 model (1.1), relating the deconfinement strategy adopted by Moroccan authorities with two scenarios. We assume $u$ defined as follows:

$$
u= \begin{cases}u_{0}, & \text { on [March 2, March 10]; } \\ u_{1}, & \text { on (March 10, March 16]; } \\ u_{2}, & \text { on (March 16, March 20]; } \\ u_{3}, & \text { on (March 20, April 6]; } \\ u_{4}, & \text { on (April 6, April 25]; } \\ u_{5}, & \text { from April 25 on; }\end{cases}
$$

where $u_{i} \in(0,1]$, for $i=0,1,2,3,4,5$, measures the effectiveness of applying the multiple preventive interventions imposed by the authorities and presented in Table 1 .

COVID-19 is known as a highly contagious disease and its transmission rate, $\beta$, varies from country to country, according to the density of the country and movements of its population. Ozair et al. [17] assumed $\beta$ to be [0.198 - 0.594] per day for Romania, and [0.097 - 0.291] per day for Pakistan. Further, Kuniya [10] estimated $\beta$ as $0.26(95 \% C I, 2.4-2.8)$. Observing the number of daily reported cases of COVID-19 in Morocco, we estimate $\beta$ as $0.4517(95 \% C I, 0.4484-0.455)$. After the infection, the patient remains in a latent period for 5.5 days $[1,20]$, in average, before becoming symptomatic and infectious or asymptomatic with a percentage that varies from $20.6 \%$ of infected population to $39.9 \%$ [15], while the time needed before his hospitalization is estimated to be 7.5 days $[6,8,19]$. All the parameter values chosen for the D-COVID-19 model (1.1) are summarized in Table 2.

Remark 4.1. From a biological point of view, the latency period is independent of the region or country under study, depending only on the structural nature of the SARS-CoV-2 coronavirus. 


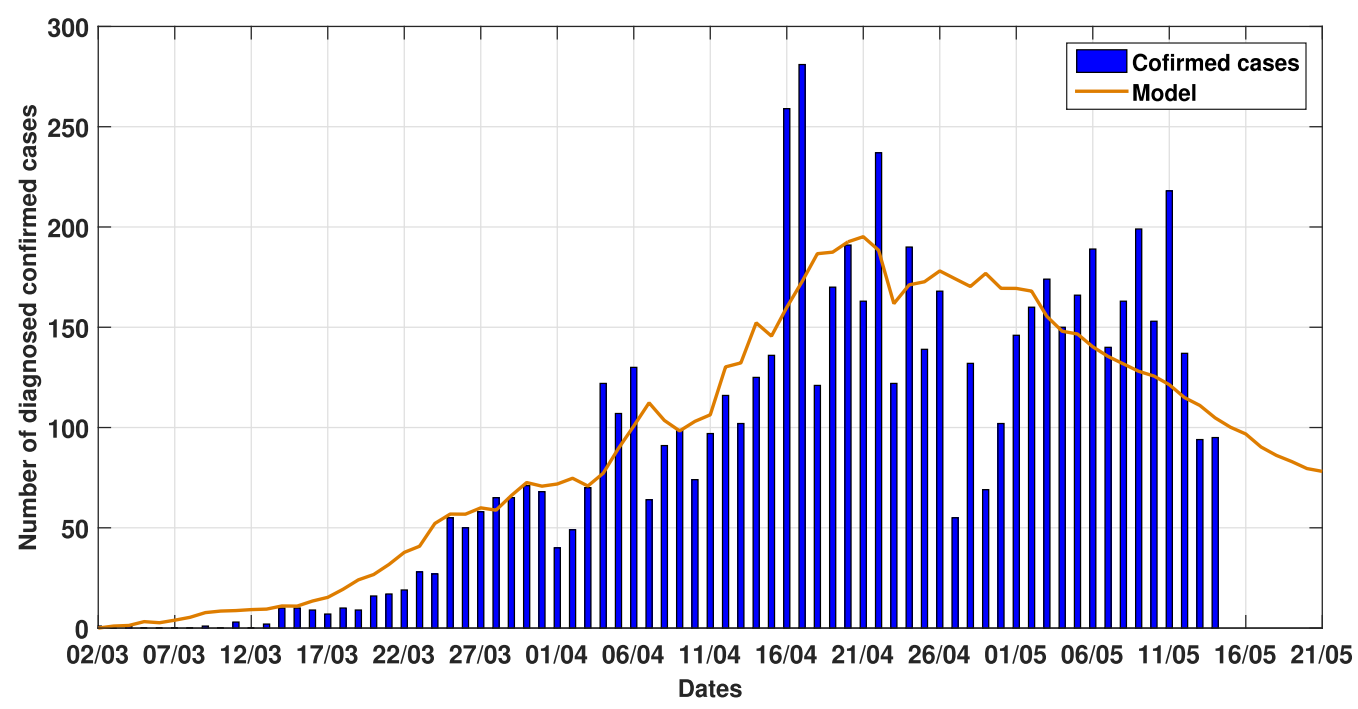

FiguRE 2. Evolution of COVID-19 confirmed cases in Morocco per day: curve predicted by our model (1.1) accordigly with Tables 1 and 2 versus real data.
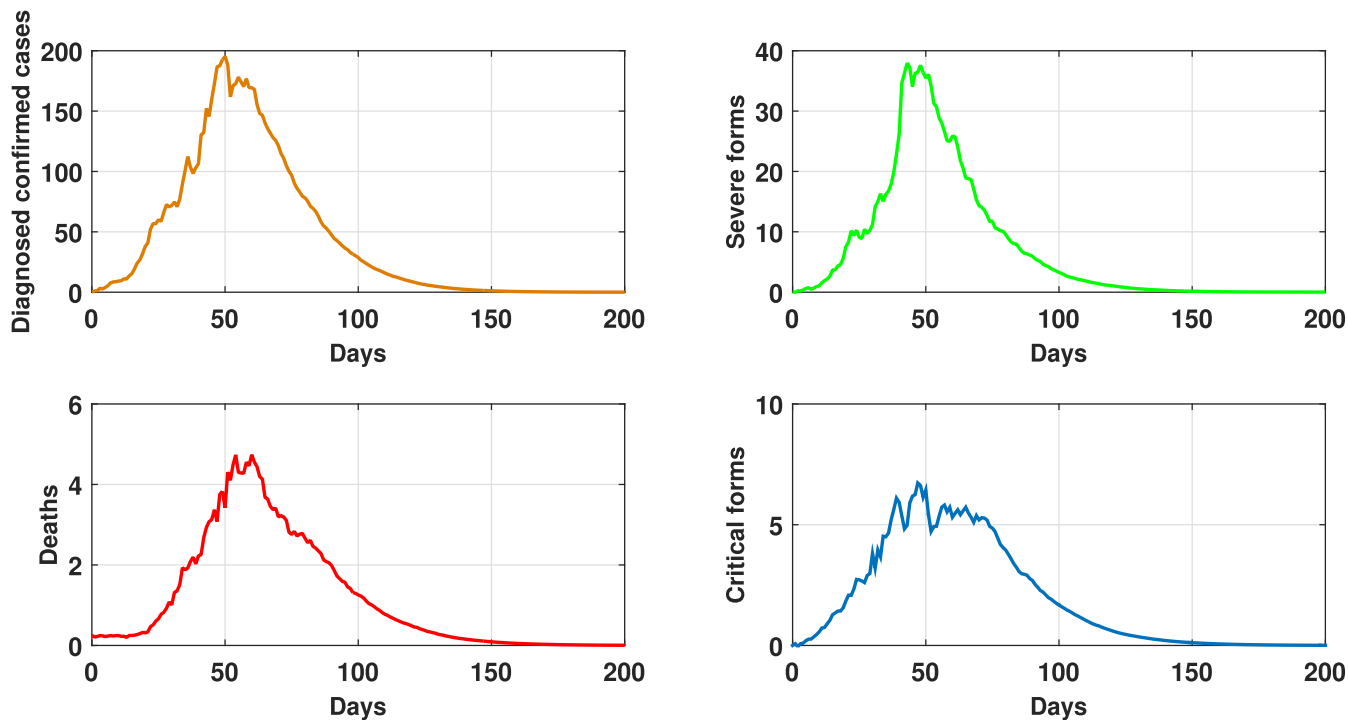

Figure 3. Evolution given by the D-COVID-19 model (1.1) without deconfinement $(\rho=0)$.

We consider that all measures and the adopted confinement strategy previously discussed are conserved. The evolution on the number of diagnosed infected positive individuals given by the D-COVID-19 model (1.1) versus the daily reported confirmed cases of COVID-19 in Morocco, from March 2 to May 6, is presented in Figure 2. We see that the curve generated by the D-COVID-19 model (1.1) follows the trend of the daily reported cases in Morocco. So, we confirm that the implemented measures taken by the authorities have an explicit impact on the propagation of the virus in the population since the curve of the D-COVID-19 model (1.1) has been flattening from April 17 and tends to go towards the extinction of the disease from May 05. In Figure 3, we see that Morocco has spent almost $40 \%$ of the total duration of the epidemic at May 11 and will reach extinction after four months, in average, from the start of the epidemic on March 2, $2020(t=0)$. 


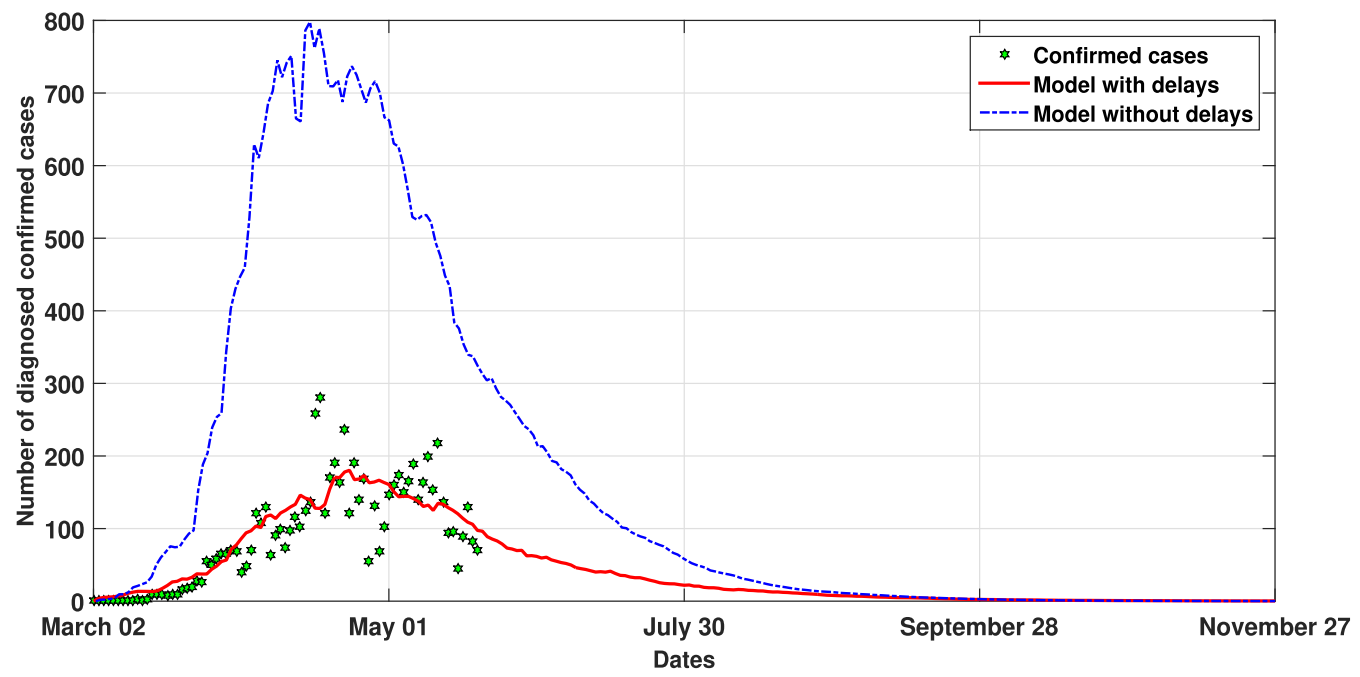

Figure 4. Effect of delays on the diagnosed confirmed cases versus clinical data.
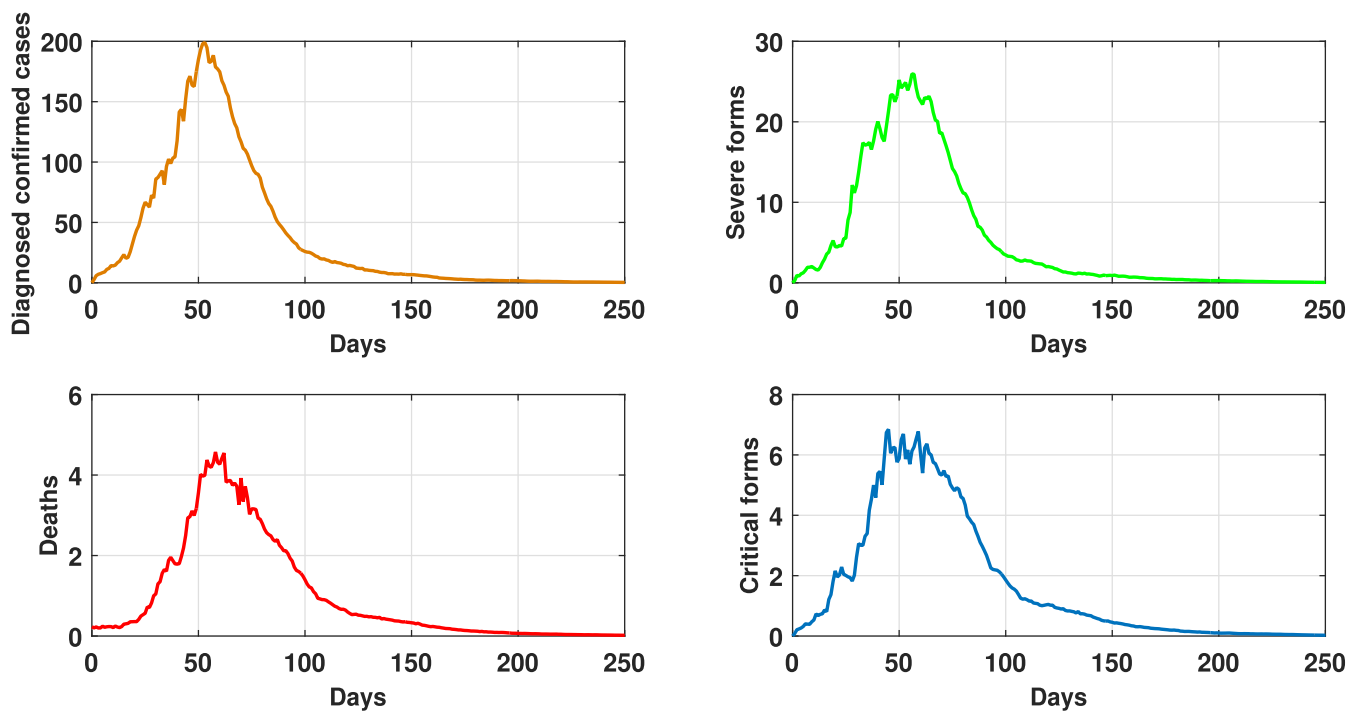

Figure 5. Evolution of the D-COVID-19 model (1.1) with deconfinement $(\rho=0.3)$ from May 20, 2020 and high effectiveness of the measures $\left(\sigma_{2}=0.01\right)$.

To prove the biological importance of delay parameters, we give the graphical results of Figure 4, which allow to compare the evolution of diagnosed positive cases with and without delays. We observe in Figure 4 a high impact of delays on the number of diagnosed positive cases. Indeed, the plot of model (1.1) without delays $\left(\tau_{i}=0, i=1,2,3,4\right)$ is very far from the clinical data. Thus, we conclude that delays play an important role in the study of the dynamical behavior of COVID-19 worldwide, especially in Morocco, and allows to better understand the reality.

In Figures 5-7, we consider the deconfinement of $30 \%$ of the population returning to work from May 20, and this proportion is immediately integrated into the susceptible population. Numerical simulations are presented for three possible scenarios. In the first, we consider that the whole population highly respects the majority of the measures announced by the authorities in relation with the deconfinement (Fig. 5). The second and third 

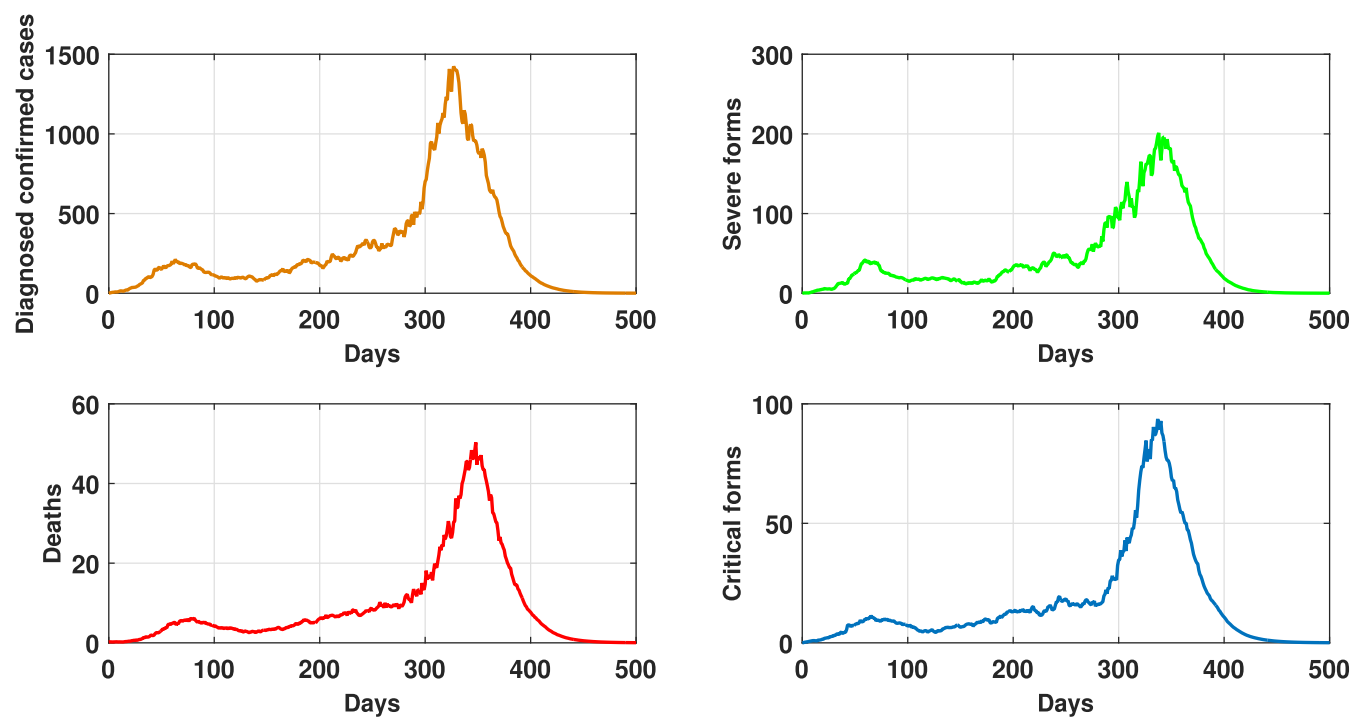

Figure 6. Evolution of the D-COVID-19 model (1.1) with deconfinement $(\rho=0.3)$ from May 20, 2020 and moderate effectiveness of the measures $\left(\sigma_{2}=0.10\right)$.
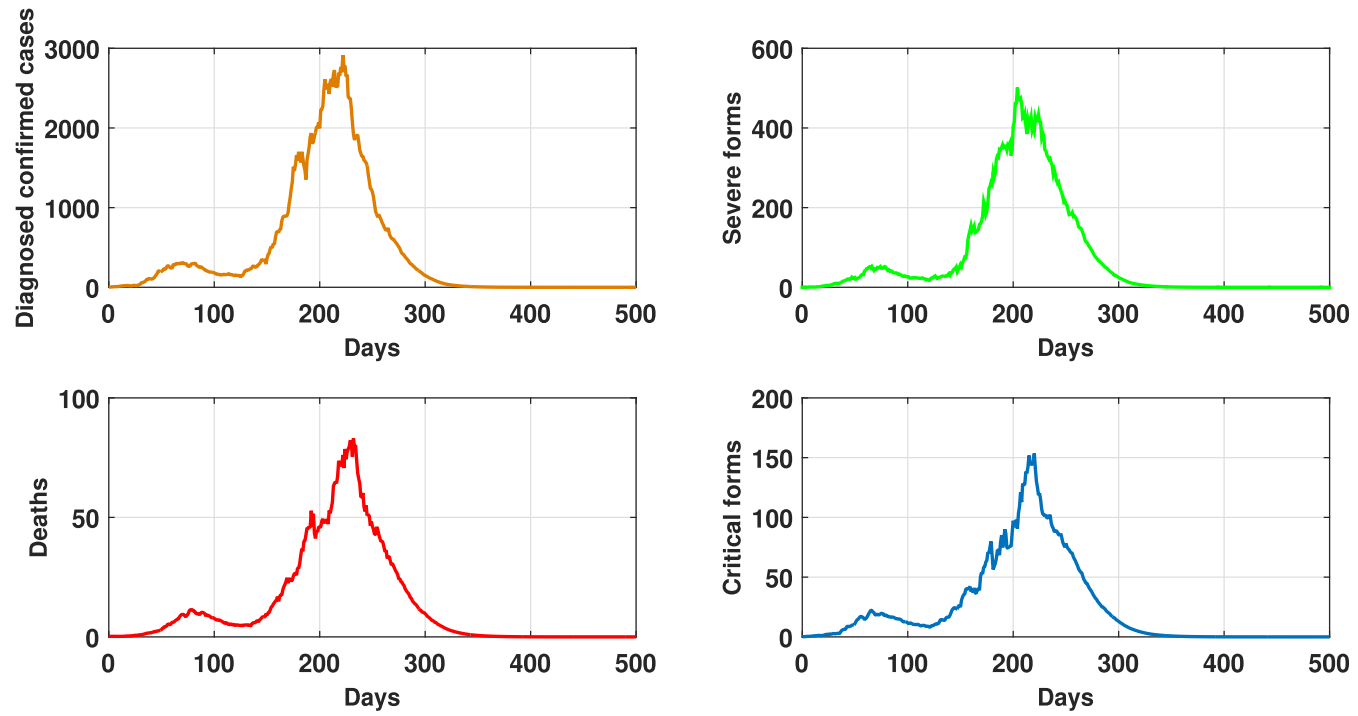

Figure 7. Evolution of the D-COVID-19 model (1.1) with deconfinement $(\rho=0.3)$ from May 20, 2020 and moderate effectiveness of the measures $\left(\sigma_{2}=0.15\right)$.

scenarios show the direct impact on the curves when the population moderately respects the measures with different levels, $\sigma_{2}=0.10$ and $\sigma_{2}=0.15$, respectively. With the last two scenarios we observe the growth in the final number of infected, deaths, severe and critical forms, which are the most important to monitor, since the health system should not be saturated. It is also important to note the appearance of a second significant peak and the fact that the time required for extinction becomes longer, which relates to the value of $\sigma_{2}$ (Figs. 6 and 7). 
It should be mentioned that all our plots were obtained using the Matlab numerical computing environment by discretizing system (1.1) by means of the higher order method of Milstein presented in [7] and used in [11].

\section{Conclusion}

In this work, we have proposed a delayed stochastic mathematical model to describe the dynamical spreading of COVID-19 in Morocco by considering all measures designed by authorities, such as confinement and deconfinement policies. More precisely, our model takes into account four types of delays: the first one is related to the incubation period, the second is the time needed to move from the symptomatic infected individuals to the three forms of diagnosed cases, the third is the time needed to move from the class of infected individuals to the recovered or dead class, while the last one is the time needed to pass from the three types of classes of individuals supported by the Moroccan health system, and under quarantine, to the recovered or dead compartments. Besides, to well describe reality, we have added a stochastic factor resulting from possible maladjustment of the population individuals to the measures.

To show that our model is mathematically and biologically well-posed, we have proved the global existence of a unique positive solution (see Thm. 2.1). Our result has shown a possible extinction of the disease when $\sigma_{1}^{2}$ is greater than a threshold parameter (see Thm. 3.1).

In addition, numerical simulations have been performed to forecast the evolution of COVID-19. More precisely, we have shown that the evolution of our D-COVID-19 model follows the tendency of daily reported confirmed cases in Morocco (see Fig. 2). Further, if Moroccan people would maintain, strictly, their confinement policy, we observe that the disease dies out around four months from March 2, 2020 (see Fig. 3). On the other hand, in response to the decision of deconfinement represented by the liberation of the $30 \%$ of population, which took place at May 20, we simulate three scenarios corresponding to different values of the intensity $\sigma_{2}$. When $\sigma_{2}=0.01$ (Fig. 5), the eradication of the disease from the population comes early compared to the cases when $\sigma_{2}=0.10$ (Fig. 6) and $\sigma_{2}=0.15$ (Fig. 7). Additionally, the number of diagnosed confirmed cases mainly changes because of the value of this intensity and a small perturbation leads to relevant quantitative changes and significant variations on the time needed for extinction. Thus, we observe that the value of this perturbation has a high impact on the evolution of COVID-19, which means the Moroccan population has a big interest to respect the governmental measures announced May 20, 2020, in order to have a successful and good deconfinement strategy.

Here we have compared the predictions of the proposed model (1.1) with real data until middle of May 2020. We leave the comparison of the real data in Morocco till the end of 2020 to a future work, where we also plan to incorporate the predictions of the evolution of our COVID-19 model with respect to preventive Moroccan measures by regions and cities.

Acknowledgements. The authors are grateful to an Associate Editor and two anonymous referees, who kindly reviewed an earlier version of the manuscript and provided several valuable suggestions and comments.

\section{REFERENCES}

[1] S.G. Baum, COVID-19 incubation period: An update, Journal Watch. Available from https://www.jwatch.org/na51083/ 2020/03/13/covid-19incubation-period-update (2020).

[2] M.S. Boudrioua and A. Boudrioua, Predicting the COVID-19 epidemic in Algeria using the SIR model. Preprint medRxiv https://doi.org/10.1101/2020.04.25.20079467 (2020).

[3] D. Fanelli and F. Piazza, Analysis and forecast of COVID-19 spreading in China, Italy and France. Chaos Solitons Fract. 134 (2020) 109761.

[4] A.E. Gorbalenya, S.C. Baker, R.S. Baric, R.J. de Groot, C. Drosten, A.A. Gulyaeva, B.L. Haagmans, C. Lauber, A.M. Leontovich, B.W. Neuman, D. Penzar, S. Perlman, L.L.M. Poon, D.V. Samborskiy, I.A. Sidorov, I. Sola and J. Ziebuhr, The species severe acute respiratory syndrome related coronavirus: classifying 2019-nCoV and naming it SARS-CoV-2. Nat. Microbiol. (2020) 1-9.

[5] A. Grai, D. Greenhalgh, L. Hu, X. Mao and J. Pan, A stochastic differential equations SIS epidemic model. SIAM J. Appl. Math. 71 (2011) 876-902.

[6] Haut Conseil de la Santé Publique, Avis relatif aux recommandations thérapeutiques dans la prise en charge du COVID-19 (complémentaire A l'avis du 5 mars 2020). Available from: https://sfar.org/ avis-relatif-aux-recommandations-therapeutiques-dans-la-prise-en-charge-du-covid-19/ (2020). 
[7] D.J. Higham, An algorithmic introduction to numerical simulation of stochastic differential equations. SIAM Rev. 43 (2001) $525-546$.

[8] C. Huang, Y. Wang, X. Li, L. Ren, J. Zhao, Y. Hu, L. Zhang, G. Fan, J. Xu, X. Gu, Z. Cheng, T. Yu, J. Xia, Y. Wei, W. Wu, X. Xie, W. Yin, H. Li, M. Liu, Y. Xiao, H. Gao, L. Guo, J. Xie, G. Wang, R. Jiang, Z. Gao, Q. Jin, J. Wang and B. Cao, Clinical features of patients infected with 2019 novel coronavirus in Wuhan, China. Lancet 395 (2020) $497-506$.

[9] B. Ivorra, M.R. Ferrandez, M. Vela-Perez and A.M. Ramos, Mathematical modeling of the spread of the coronavirus disease 2019 (COVID-19) taking into account the undetected infections. The case of China. Commun. Nonlinear Sci. Numer. Simul. 88 (2020) 105303.

[10] T. Kuniya, Prediction of the epidemic peak of coronavirus disease in Japan, 2020. J. Clin. Med. 9 (2020) 789.

[11] M. Mahrouf, K. Hattaf and N. Yousfi, Dynamics of a stochastic viral infection model with immune response. MMNP 12 (2017) 15-32.

[12] X. Mao, Stochastic differential equations and applications, second edition. Horwood Publishing Limited, Chichester, (2008).

[13] Ministry of Health of Morocco, Department of Epidemiology and Disease Control. Available from: http://www.sante.gov. ma/Pages/Accueil.aspx (2020).

[14] Ministry of Health of Morocco, The official portal of Corona virus in Morocco. Available from: http://www.covidmaroc.ma/ pages/Accueil.aspx (2020).

[15] K. Mizumoto, K. Kagaya, A. Zarebski and G. Chowell, Estimating the asymptomatic proportion of coronavirus disease 2019 (COVID-19) cases on board the Diamond Princess cruise ship, Yokohama, Japan, 2020. Euro Surveill. 25 (2020) 2000180.

[16] F. Ndaïrou, I. Area, J.J. Nieto and D.F.M. Torres, Mathematical modeling of COVID-19 transmission dynamics with a case study of Wuhan. Chaos Solitons Fract. 135 (2020) 109846.

[17] M. Ozair, T. Hussain, M. Hussain, A.U. Awan and D. Baleanu, Estimation of transmission potential and severity of COVID-19 in Romania and Pakistan. Preprint medRxiv https://doi.org/10.1101/2020.05.02.20088989 (2020).

[18] B. Tang, X. Wang, Q. Li, N. L. Bragazzi, S. Tang, Y. Xiao and J. Wu, Estimation of the transmission risk of the 2019-nCoV and its implication for public health interventions. J. Clin. Med. 9 (2020) 462.

[19] D. Wang, B. Hu, C. Hu, F. Zhu, X. Liu, J. Zhang, B. Wang, H. Xiang, Z. Cheng, Y. Xiong, Y. Zhao, Y. Li, X. Wang and Z. Peng, Clinical characteristics of 138 hospitalized patients with 2019 novel coronavirus-infected pneumonia in Wuhan, China. JAMA 323 (2020) 1061-1069.

[20] WHO, Coronavirus disease 2019 (COVID-19) Situation Report 73. Available from: https://apps.who.int/iris/handle/10665/ $331686(2020)$

[21] J.T. Wu, K. Leung and G.M. Leung, Nowcasting and forecasting the potential domestic and international spread of the 2019-nCoV outbreak originating in Wuhan, China: a modelling study. The Lancet 395 (2020) 689-697. 